\title{
ACTIVATION OF COAGULATION AND FIBRINOLYTIC PATHWAYS IN PATIENTS WITH LEFT VENTRICULAR ASSIST DEVICES
}

Talia Spanier, MD $^{a}$ Mehmet Oz, $\mathrm{MD}^{\mathrm{a}}$

Howard Levin, $\mathrm{MD}^{\mathrm{b}}$

Alan Weinberg, MS ${ }^{\mathrm{c}}$

Kathy Stamatis, MS ${ }^{c}$

David Stern, MD ${ }^{\text {a,d }}$

Eric Rose, MD ${ }^{\mathrm{a}}$

Ann Marie Schmidt, MD ${ }^{a, b}$
Left ventricular assist devices have provided successful supportive therapy for patients awaiting cardiac transplantation for extended periods of time. Although thromboembolic events have complicated support with these devices, the HeartMate left ventricular assist device developed by Thermo Cardiosystems, Inc., Woburn, Massachusetts, was specifically designed with a textured blood-contacting surface to minimize this risk. Clinical experience with this device has been encouraging, inasmuch as minimal thromboembolic complications have occurred despite the absence of anticoagulation. The coagulation and fibrinolytic pathways in these individuals were investigated to better understand the hematologic status of patients treated with the Thermo Cardiosystems device. Despite apparently normal prothrombin and activated partial thromboplastin times, as well as platelet counts, evidence of significant thrombin generation and fibrinolysis was present. To eliminate underlying cardiac failure as the responsible factor for these abnormalities, we made similar measurements in patients with end-stage heart failure who were not supported by an assist device or anticoagulation. These measurements revealed no evidence of thrombin generation or fibrinolysis. These data demonstrate that patients supported with a left ventricular assist device, while successfully sustained without systemic anticoagulation, nevertheless have evidence of activation of coagulation. These phenomena appear to be related to the presence of the device rather than to the underlying cardiac abnormalities. Although procoagulant and fibrinolytic pathways are apparently balanced in these patients, these data underscore the potential for the development of bleeding or thrombosis in clinically relevant settings. (J Thorac Cardiovasc Surg 1996; 112:1090-7)
C ardiac transplantation has become the accepted standard of care for patients with end-stage heart failure. The ever-widening gap between the number of potential cardiac transplant recipients and the

From the Departments of Surgery, ${ }^{\mathrm{a}}$ Medicine, ${ }^{\mathrm{b}}$ Biostatistics, ${ }^{\mathrm{c}}$ and Physiology, ${ }^{\mathrm{d}}$ Columbia University College of Physicians and Surgeons, New York, N.Y.

Supported by grants from the U.S. Public Health Service (AG00602), the American Heart Association (New York affiliate), and The American Association for Thoracic Surgery (Evarts A. Graham Traveling Fellowship). Dr. Oz is an Irving Scholar at Columbia University.

Received for publication Jan. 18, 1996; revisions requested March 5, 1996; revisions received May 22, 1996; accepted for publication May 23, 1996.

Address for reprints: Talia Spanier, MD/Ann Marie Schmidt, MD, Columbia University College of Physicians and Surgeons, 630 W. 168 St., P\&S 11-518, New York, NY 10032.

Copyright (C) 1996 by Mosby-Year Book, Inc.

$0022-5223 / 96 \$ 5.00+0 \quad \mathbf{1 2 / 1 / 7 5 4 4 3}$ number of available donor organs, ${ }^{1-3}$ however, has necessitated the development of intermediate or "bridging" devices designed to sustain the patient awaiting an appropriate donor heart. The success of these pumps has prompted a movement toward their use as a permanent form of cardiac replacement. ${ }^{4-8}$ One of the critical factors limiting the use of left ventricular assist devices (LVADs) in the past has been a reported rate of thromboembolic complications of $30 \%$ or greater, as well as a significant perioperative coagulopathy. ${ }^{9-12}$

Compared with the early LVADs, whose surfaces were mainly smooth, the HeartMate LVAD developed by Thermo Cardiosystems, Inc., Woburn, Massachusetts, was designed with specific features intended to minimize the potential for thromboembolic complications. ${ }^{13-17}$ Specifically, this LVAD, containing a textured surface, was designed to facilitate the formation of a tightly adherent "pseudo- 
Table 1. Demographic profile of patients having LVADs

\begin{tabular}{rrrrrc}
\hline & $\begin{array}{c}\text { Age } \\
(y r)\end{array}$ & Sex & Diagnosis & $\begin{array}{c}\text { Length of LVAD } \\
\text { treatment (days) }\end{array}$ & $\begin{array}{c}\text { Type of } \\
\text { LVAD pump* }\end{array}$ \\
\hline 1 & 52 & $\mathrm{~F}$ & Idiopathic CM & 335 & Pneumatic \\
2 & 65 & $\mathrm{M}$ & Ischemic CM & 46 & Pneumatic \\
3 & 51 & $\mathrm{M}$ & Ischemic CM & 139 & Pneumatic \\
4 & 19 & $\mathrm{M}$ & Idiopathic CM & 35 & Electric \\
5 & 61 & $\mathrm{M}$ & Ischemic CM & 101 & Electric \\
6 & 17 & $\mathrm{M}$ & Idiopathic CM & 65 & Electric \\
7 & 61 & $\mathrm{M}$ & Ischemic CM & 100 & Electric \\
8 & 58 & $\mathrm{M}$ & Ischemic CM & 70 & Electric \\
9 & 58 & $\mathrm{M}$ & Ischemic CM & 68 & Pneumatic \\
10 & 48 & $\mathrm{M}$ & Ischemic CM & 521 & Electric \\
11 & 68 & $\mathrm{M}$ & Ischemic CM & 49 & Pneumatic \\
12 & 39 & $\mathrm{~F}$ & Subacute MI & 9 & Pneumatic \\
13 & 56 & $\mathrm{M}$ & Ischemic CM & 7 & Pneumatic \\
14 & 51 & $\mathrm{~F}$ & Subacute MI & 33 & Pneumatic \\
15 & 65 & $\mathrm{M}$ & Ischemic CM & 35 & Electric \\
16 & 59 & $\mathrm{~F}$ & Ischemic CM & 33 & Pneumatic \\
17 & 65 & $\mathrm{M}$ & Ischemic CM & 25 & Pneumatic \\
18 & 52 & $\mathrm{M}$ & Idiopathic CM & 11 & Pneumatic \\
19 & 51 & M & Ischemic CM & 5 & Pneumatic \\
20 & 30 & M & Idiopathic CM & & Pneumatic \\
\hline
\end{tabular}

$C M$, Cardiomyopathy; $M I$, myocardial infarction.

"HeartMate LVAD; Thermo Cardiosystems, Inc., Woburn, Massachusetts.

neointima" on the blood-contacting surfaces, a strategy aimed at reducing the risk of thromboembolism by eliminating direct contact between the device and circulating blood. ${ }^{17} \mathrm{~A}$ thromboembolic rate of less than $2 \%$ has been associated with the use of this device in patients receiving no or minimal forms of anticoagulation. ${ }^{18}$

To better understand the hematologic profile that underlies this low thromboembolic risk and to more fully understand the long-term physiologic implications of its use, we analyzed measurements of thrombin generation and fibrinolysis in patients supported by the HeartMate LVAD.

\section{Patients and methods}

Patients. Twenty patients in clinically stable condition with implanted Thermo Cardiosystems HeartMate LVADs and 12 other patients in clinically stable condition with New York Heart Association class IV heart failure were entered into this study during the period extending from June 1995 through November 1995. The study was conducted in accordance with the rules and regulations of the Institutional Review Board of Columbia University College of Physicians and Surgeons. Among the patients supported with an LVAD, 14 had pneumatically driven LVADs and the other six had electrically driven LVADs. The mean age of the patients with an LVAD was 51.7 years (range 17 to 65 years) and the mean duration of LVAD treatment was 83 days (range 5 to 335 days). No patients in the LVAD group were receiving heparin, warfarin sodium, or other systemic anticoagulant therapy.
Five patients were receiving aspirin $(325 \mathrm{mg}$ by mouth every day) (Table I). The 12 patients with class IV end-stage heart failure were selected for inclusion in this study because they were not receiving systemic anticoagulation. Their mean age was 54 years (range 33 to 65 years). All patients in this group were receiving aspirin (325 mg by mouth every day).

Values for a normal control population were obtained from the clinical laboratories at Columbia Presbyterian Medical Center for prothrombin time, activated partial thromboplastin time, levels of platelets and fibrinogen, fibrinogen (fibrin) degradation products (FDPs), and D-dimers. These values were obtained by examining blood samples of 30 normal volunteers. Normal values for thrombin-antithrombin complex (TAT), prothrombin activation peptide $\left(\mathrm{F}_{1+2}\right)$, and soluble thrombomodulin were provided by the manufacturers from which the enzyme-linked immunosorbent assay (ELISA) kits were obtained. These values were derived by analysis of plasma samples from 120 clinically stable volunteers. Levels of $F_{1+2}$ are a specific reflection of the effects of factor $\mathrm{Xa}$ on prothrombin and thus are a marker of thrombin generation and activation of coagulation. ${ }^{19-20}$

Materials. After peripheral blood had been obtained in accordance with the aforementioned protocols, complete blood count, prothrombin time, activated partial thromboplastin time, fibrinogen levels, and FDPs were obtained from the clinical laboratories of Columbia University College of Physicians and Surgeons according to standard methods. ELISAs for TAT and $F_{1+2}$ were obtained from Behring Diagnostics, Inc. (Westwood, Mass.), and ELISA kits for D-dimers and soluble thrombomodulin were obtained from American Diagnostica (Greenwich, 
A. PROTHROMBIN TIME

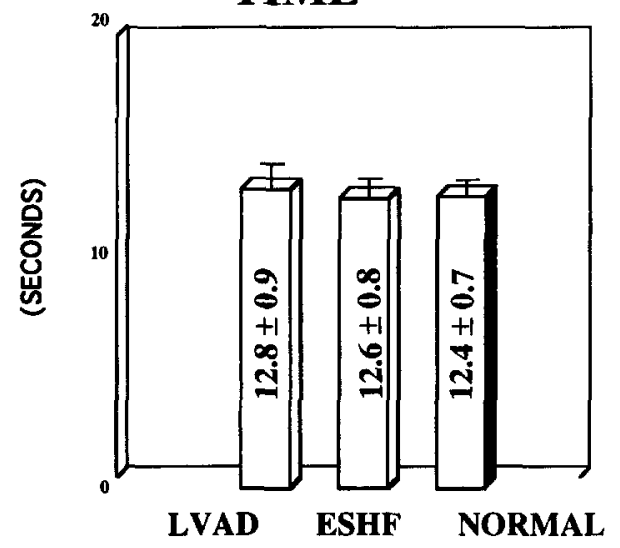

C. Platelets

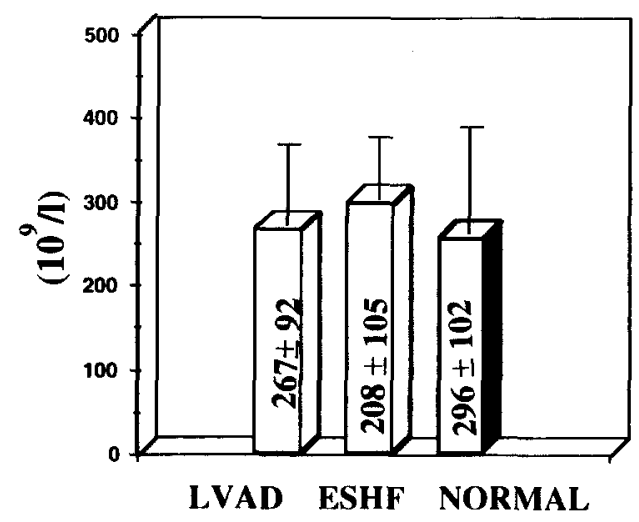

\section{B. PARTIAL THROMBOPLASTIN TIME}

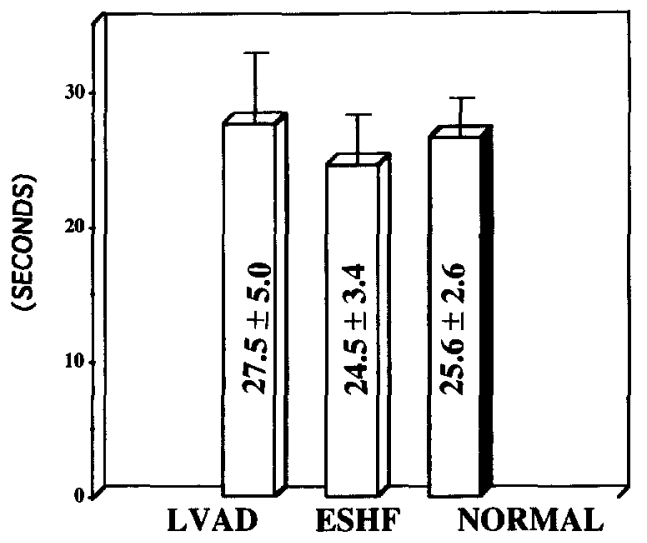

D. Fibrinogen

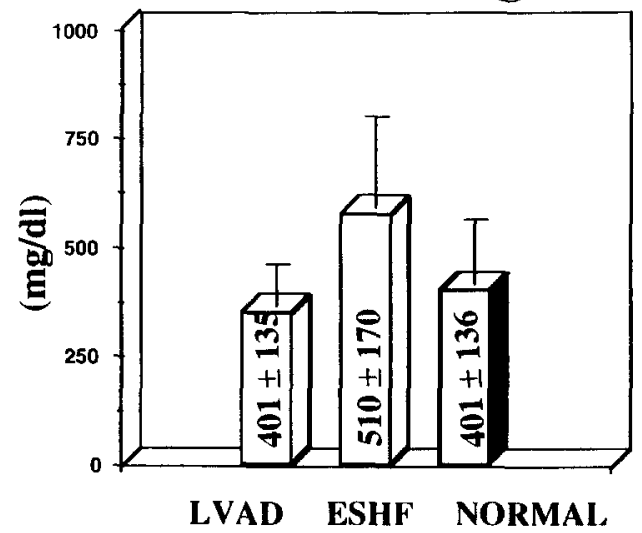

Fig. 1. Assessment of routine coagulation parameters in patients with LVADs and patients with end-stage heart failure $(E S H F)$. Plasma was obtained by peripheral venipuncture from the patients with LVADs or end-stage heart failure as described in the text. Parameter (normal control) values were obtained from the clinical coagulation laboratory of Columbia Presbyterian Medical Center. Mean values \pm standard deviation for prothrombin time, activated partial thromboplastin time, and levels of platelets and fibrinogen are depicted within the bar graphs. No significant differences were noted except that patients with end-stage heart failure had higher levels of fibrinogen than patients with LVADs and normal control subjects $(p<0.02)$.

Conn.). All ELISAs were performed according to the manufacturers' instructions.

Statistical analysis. Data were analyzed with SAS System software (SAS Institute, Inc., Cary, N.C.). The Kruskal-Wallis test $\left(\chi^{2}\right.$ approximation $\left.{ }^{21}\right)$ and the nonparametric analog of the analysis of variance were used to test the differences in means across the three groups (LVAD, end-stage heart failure, and normal). If the Kruskal-Wallis test showed significant differences, then a nonparametric multiple comparisons test was applied to determine among which groups statistically significant differences existed. ${ }^{22,23}$ Differences were considered significant if the probability value ( $p$ value) for comparison was less than 0.05 .

\section{Results}

As previously described, plasma was obtained from patients at random times during LVAD therapy which corresponded to our study period. The mean length of LVAD implantation at the time of blood sampling was 87 days (range 5 to 335 days). The patients were all in clinically stable condition when they were tested. Blood sampling was performed in the morning in all cases, but no samples were obtained with the patient fasting. As stated previously, no patients were receiving oral or intravenous anticoagulant therapy. Five of the patients 


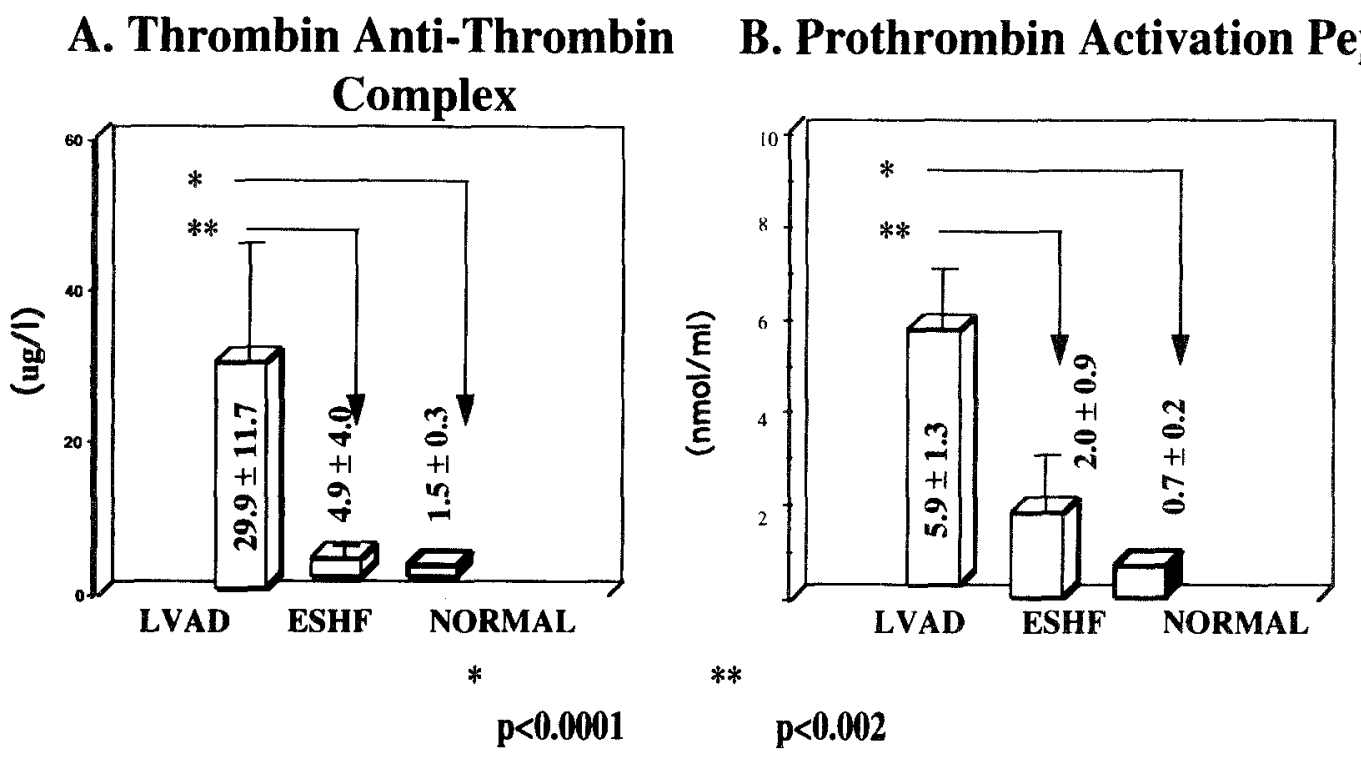

Fig. 2. Assessment of thrombin generation in patients with LVADs and in patients with end-stage heart failure ( $E S H F)$. Peripheral blood was obtained as described in the text and plasma was assayed as described for $\mathrm{F}_{1+2}$ and TAT. Mean values \pm standard deviation are shown within the bar graphs. Levels of $\mathrm{F}_{1+2}$ and TAT were significantly higher in patients with LVADs than in patients with end-stage heart failure and than the normal control values ( $p<0.002$ and $p<0.0001$, respectively).

were receiving aspirin at a dosage of $325 \mathrm{mg}$ one time a day. Normal population control values were obtained from our clinical laboratories or from the manufacturers of the respective assay kits used in these experiments. Plasma was also obtained from patients with class IV heart failure to eliminate the presence of heart failure as a cause of the observed coagulation abnormalities. These samples were similarly taken in the morning, but not with the patient fasting. All of these patients were receiving aspirin ( $325 \mathrm{mg}$ orally every day), but none was receiving anticoagulant therapy.

Our initial studies revealed that there were no significant differences between patients supported by an LVAD and the normal population with respect to platelet count, prothrombin and partial thromboplastin times, and levels of plasma fibrinogen. Comparison with patients in end-stage heart failure also failed to reveal any significant differences in any of these studies (Fig. 1).

To evaluate more subtle indices indicative of activation of the procoagulant pathway, we measured levels of TAT and $F_{1+2}$. Evaluation of these markers of thrombin generation revealed that patients supported with an LVAD had statistically significantly higher levels of TAT $(p<0.0001)$ and $\mathrm{F}_{1+2}(p<0.0001)$ than the control population (Fig.
2). Similarly, these markers of thrombin generation were significantly higher in patients with an LVAD than in patients with end-stage heart failure $(p<$ 0.002 ). There were no discernible differences between patients with pneumatically driven or electrically powered LVADs with respect to markers of thrombin generation, nor were differences detected in LVAD patients receiving aspirin compared with those receiving no antiplatelet therapy (data not shown).

To investigate whether enhanced fibrinolysis accompanied activation of the procoagulant pathway, we evaluated patients for the presence of D-dimers and FDPs. Compared with the normal control population and patients with end-stage heart failure, patients with an LVAD had significantly higher levels of plasma D-dimers and FDPs $(p<0.0001$ and $p<0.0001$, respectively) (Fig. 3). Levels of D-dimer and FDP in patients with an LVAD were similarly elevated irrespective of the type of LVAD or treatment with aspirin (data not shown).

We examined levels of soluble thrombomodulin as an additional marker of endothelial disturbance potentially caused by the effects of microemboli generated in the coagulopathy associated with LVAD therapy. Soluble thrombomodulin, most likely made and released by the endothelium, ${ }^{24}$ was 
A. D-Dimers

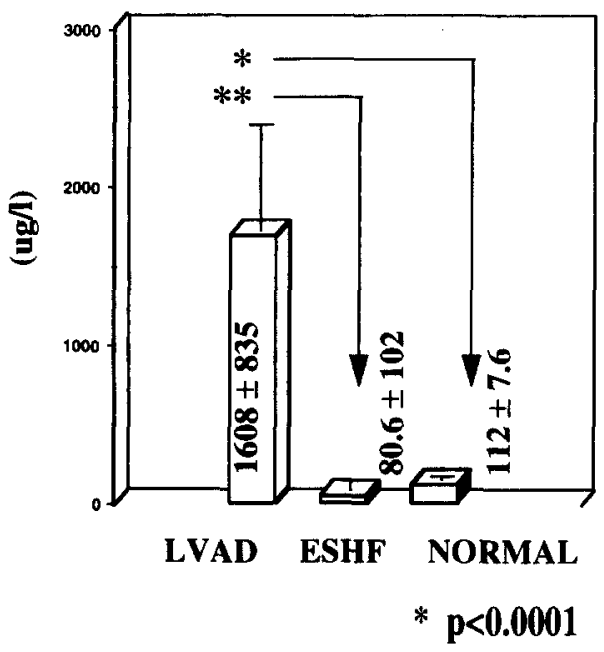

B. Fibrinogen Degradation Products

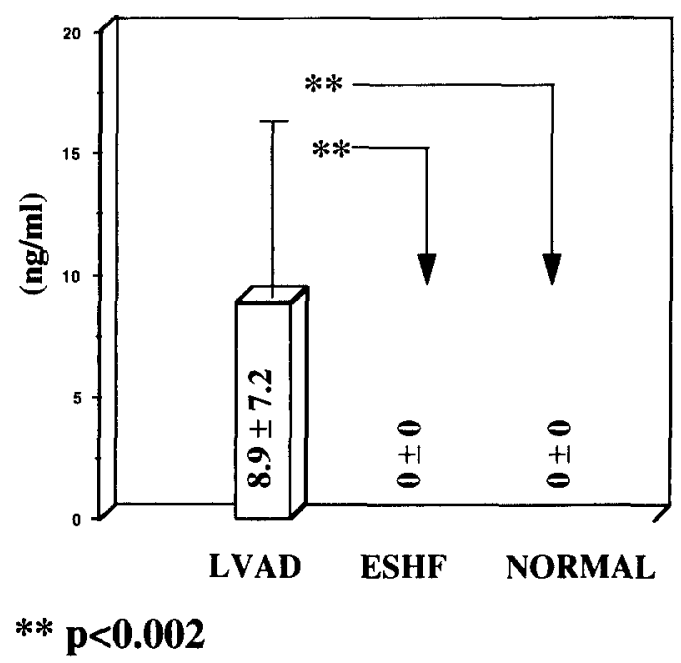

Fig. 3. Assessment of activation of fibrinolysis in patients with LVADs and patients with end-stage heart failure $(E S H F)$. Peripheral blood was obtained as described in the text and plasma was assayed as described for levels of D-dimers and FDPs. Mean values \pm standard deviation are shown within the bar graphs. Levels of D-dimers were significantly higher in patients with LVADs than in patients with end-stage heart failure and than the normal control values $(p<0.002$ and $p<0.002$, respectively). Levels of FDPs were significantly higher in patients with LVADs than in patients with end-stage heart failure and than the normal control values ( $p<0.0001$ and $p<0.0001$, respectively).

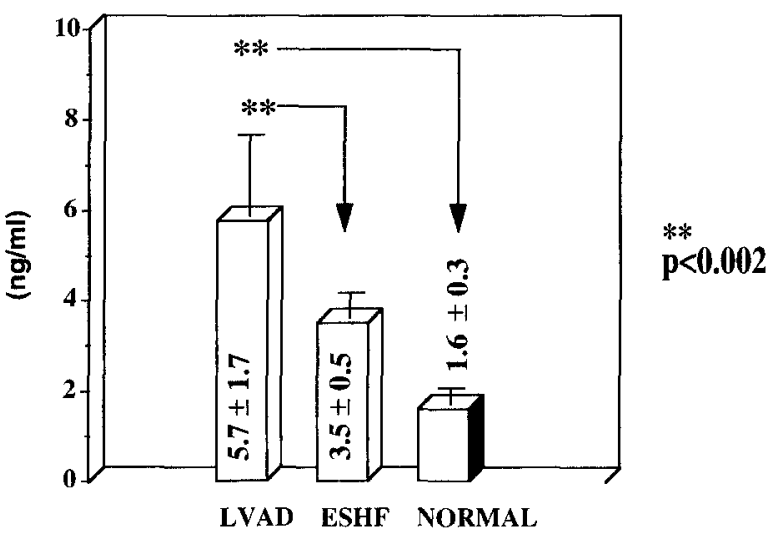

Fig. 4. Elevation of soluble thrombomodulin in patients with LVADs: a possible marker of endothelial perturbation. Peripheral blood was obtained as described in the text and plasma was assayed as described for levels of soluble thrombomodulin. Mean values of soluble thrombomodulin \pm standard deviation are shown within the bar graphs. Levels of soluble thrombomodulin were significantly higher in patients with LVADs than the normal control values $(p<0.002)$. There was no significant difference in levels of soluble thrombomodulin in patients with LVADs and patients with end-stage heart failure sustained without anticoagulation. at a higher concentration in the plasma of patients with an LVAD than in normal subjects $(p<0.002)$ (Fig. 4). Increased plasma soluble thrombomodulin is likely to result from endothelial perturbation caused by a number of factors including sustained activation of coagulation and fibrinolytic pathways, altered blood flow, or other vascular changes consequent to the presence of the LVAD.

\section{Discussion}

Our data indicate that patients with Thermo Cardiosystems HeartMate LVADs have significant activation of coagulation with secondary fibrinolysis, despite both apparent clinical stability and "normal" screening values of routine hemostatic parameters such as platelet count, prothrombin time, and activated partial thromboplastin time. Soluble thrombomodulin, derived from an integral membrane protein constitutively expressed by endothelium, ${ }^{24}$ is likely to be shed or proteolyzed as a result of endothelial perturbation, perhaps related to the presence of the LVAD or to microemboli associated with activation of coagulation (Fig. 5). From a clinical standpoint, the extent of activation of the 

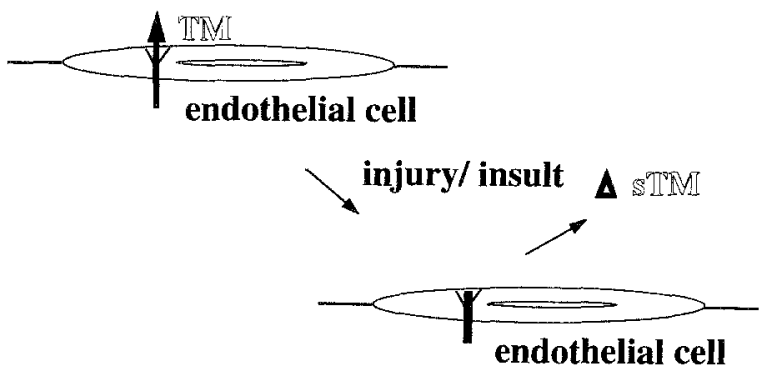

Fig. 5. Soluble thrombomodulin. We postulate that soluble thrombomodulin, likely derived from the integral membrane protein constitutively expressed by the endothelium, is shed and/or proteolyzed as a result of endothelial perturbation caused by the presence of the LVAD. Such inciting causes as the presence of microemboli in a procoagulant milieu, altered flow characteristics with cellular activation, and/or other vascular factors associated with the LVAD itself are likely contributory factors.

thrombin-generating and fibrinolytic pathways appears balanced in these patients; our earlier studies demonstrated low (2\%) thromboembolic complication rates associated with the use of this LVAD. ${ }^{18}$ Furthermore, clinical experience does not demonstrate a bleeding or thrombotic diathesis in the nonstressed or nonsurgical setting. ${ }^{4-8,14-18}$

The causes underlying these hemostatic abnormalities are of considerable interest. Such LVADspecific causes as the textured polyurethane surface, the titanium housing, and the Dacron inflow/outflow lines need to be considered. Other causes, such as altered flow characteristics and the presence of preexisting clots in the diseased ventricles, must also be investigated. Our data do not suggest that these findings are a direct result of the surgical implantation procedure of the LVAD, inasmuch as hemostatic findings remain abnormal at least up to day 335 of LVAD therapy (see Figs. 1 to 4 ). In this context, Livingston and colleagues ${ }^{25}$ have shown that in the perioperative period, patients with an LVAD consistently have significantly higher markers of thrombin generation (TAT and $\mathrm{F}_{1+2}$ ) and fibrinolysis (D-dimers and FDPs) than do patients who have had coronary bypass grafting. ${ }^{25}$ Our pilot studies, in which we prospectively examined patients having LVADs, do not demonstrate that these perioperative findings are sustained, because the overt indices of thrombin generation and fibrinolysis are acute and procedure-related. In fact, pilot studies in two patients examined to date have revealed that markers of thrombin generation/fibrinolysis are significantly elevated during the operation, return to

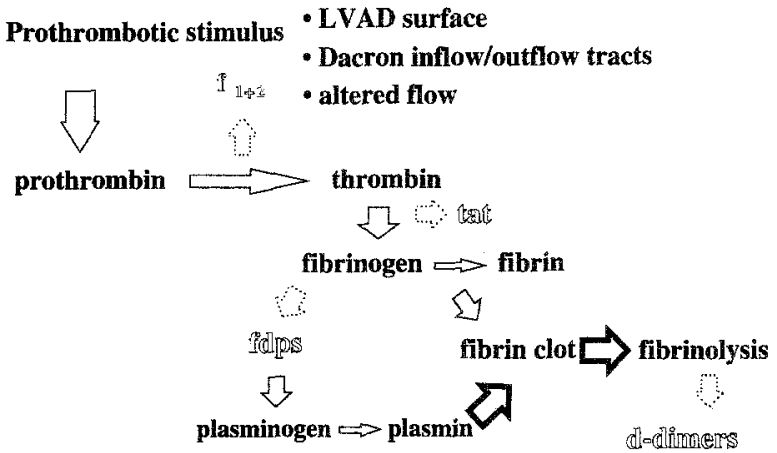

Fig. 6. Coagulation cascade/fibrinolysis. As demonstrated by our studies, evidence exists for significant thrombin generation (as reffected by increased levels of TAT and $F_{1+2}$ ) and fibrinolysis (increased levels of Ddimers and FDPs) in patients with LVADs compared with values for the normal control population and values for patients with end-stage heart failure not supported by LVAD or anticoagulant therapy. The mechanism(s) underlying the generation of a procoagulant state are not yet clear. For example, it is not known whether activation of the intrinsic and/or extrinsic pathways of coagulation underlie these findings. Closed arrows are indicative of the procoagulant pathway resulting in the formation of a fibrin clot. Open arrows follow the fibrinolytic pathway. Dotted arrows and open font signify the markers of these pathways reported in this study.

near-normal values by days 5 to 7 after the operation, and then rise in a time-dependent manner, peaking at approximately day 35 of LVAD therapy, an effect that is subsequently sustained (data not shown).

Indeed, these data suggest that a specific characteristic of the LVAD itself is responsible, at least in part, for these observations. In this context, we and others have demonstrated considerable cellular entrapment by this device in studies of explanted LVADs. ${ }^{26-30}$ Flow cytometry studies have demonstrated that the majority of these cells are myeloid/ monocytic in origin. In addition, a smaller percentage of the cells are pluripotential hematopoietic cells, which can be induced to differentiate in culture to mature hematopoietic cells. ${ }^{30}$ Menconi and colleagues $^{29}$ found that the surface of explanted LVADs contained adherent mononuclear cells, platelets, and myofibroblasts intermingled with areas of compact fibrinous material, as well as areas of collagenous tissue. Similarly, Salih and colleagues ${ }^{26}$ found the surface of the explanted LVAD polyurethane membrane to consist of fibrinous and cellular layers. The layer atop the membrane was composed 
of a compact fibrin coating, as well as numerous mononuclear cells and spindle-shaped cells. An intermediate middle layer consisted of cells resembling fibroblasts and fewer mononuclear cells than were observed in the inner layer. The outermost layer, at the biomaterial/tissue interface, contained a foreign body reaction with numerous multinucleated giant cells. Sections of tissue islands removed from the titanium surface revealed organized fibrous and collagenous tissue with few cellular areas, except for occasional mononuclear cells. Whereas Salih and colleagues found no evidence of endothelial cells on the surface of the LVAD, other studies have disputed this. Specifically, Frazier and colleagues ${ }^{27}$ found evidence for the presence of endothelial cells on the pseudointimal lining of the textured LVAD surface. Using antibodies to von Willebrand factor, they demonstrated positive immunoreactivity in certain cells on the lining, suggestive of the presence of endothelial cells. (Of course, platelet material may also be responsible for this positive immunostaining for von Willebrand factor.) The significance of defining the presence of endothelial cells in this setting cannot be overstated, because their presence may suggest the potential for restoration of normal control of the vascular procoagulant and anticoagulant pathways.

Consistent with the hypothesis that the LVAD surface itself contributes to the multiple biologic phenomena observed in the course of its use, studies have revealed that this lining is metabolically active. Menconi and associates ${ }^{29}$ showed by ribonucleic acid hybridization analysis that the colonizing cells actively expressed genes encoding proteins for cell proliferation markers, cell adhesion molecules, cytoskeletal structures, and extracellular matrix components.

Taken together, these data suggest that the cells lining the LVAD surface are not biologically inert. In this context, we postulate that the mononuclear cells, platelets, and pluripotential stem cells initially trapped by the polyurethane surface, titanium housing, or Dacron grafts ${ }^{25-30}$ may become activated, thus leading to the generation of a local proinflammatory/procoagulant state. We further postulate that this mechanism is responsible, at least in part, for triggering and subsequently sustaining activation of the coagulation and fibrinolytic cascades (Fig. 6). In fact, our recent pilot studies have demonstrated that peripheral blood-derived mononuclear cells adhere almost immediately to representative sections of the LVAD polyurethane surface and that, within days, these mononuclear cells appear to differentiate into mononuclear phagocyte-type cells with epithelioid changes consistent with their activation. Further studies are ongoing to demonstrate whether these cells produce mediators such as cytokines and tissue factor, which could certainly contribute to the initiation and propagation of procoagulant events, culminating in the generation of thrombin.

In conclusion, patients with the Thermo Cardiosystems HeartMate 1000 IP LVAD have evidence of enhanced thrombin generation with fibrinolysis. Thus, despite the apparent clinical stability of patients having an LVAD, as well as a low incidence of clinically apparent or subclinical microemboli, our results identify the presence of a coagulopathy with the potential to exacerbate bleeding or clotting complications in clinically relevant settings, for example, operation, infection, or inflammatory stress. The current balance appears to result in a favorably low thromboembolic rate; however, studies to determine the mechanisms triggering perturbation of the procoagulant and fibrinolytic pathways in patients having an LVAD are essential to identify targets of potential therapeutic intervention in these patients if necessitated by the development of hemostatic complications associated with long-term use of this device.

\section{REFERENCES}

1. Evans RW, Orians CE, Ascher NL. The potential supply of organ donors: an assessment of the efficacy of organ procurement effort in the United States. JAMA 1992;267:239-46.

2. Orians CE, Evans RW, Ascher NL. Estimates of organspecific donor availability for the United States. Transplant Proc 1993;2:25.

3. United Network for Organ Sharing (UNOS). UNOS Update 1993;9:25.

4. $\mathrm{Oz} \mathrm{M}$, Goldstein DJ, Ashton RC Jr, et al. Long term implantable left ventricular assist devices for outpatient therapy of patients with end-stage heart disease: An alternative to transplantation? Circulation 1995;92:1802.

5. McCarthy PM, James KB, Savage RM, et al. Implantable left ventricular assist device. Circulation 1994;90(5 Pt 2):II83-6.

6. Pennington DG, McBride LR, Peigh PS, Miller LW, Swartz MT. Eight years' experience with bridging to cardiac transplantation. J Thorac Cardiovasc Surg 1994;107:472-80.

7. McCarthy PM. HeartMate implantable left ventricular assist device: bridge to transplantation and future applications. Ann Thorac Surg 1995;59(suppl):s46-51.

8. Kormos RL, Murali S, Dew MA, et al. Chronic mechanical circulatory support: rehabilitation, low morbidity, and superior survival. Ann Thorac Surg 1994;57:51-8.

9. Icenogle TB, Smith RG, Cleavinger M, et al. Thromboembolic complications of the Symbion AVAD system. Artif Organs 1989;13:532-8.

10. Levinson MM, Smith RG, Cork RC, et al. Thromboembolic 
complications of the Jarvik-7 total artificial heart: case report. Artif Organs 1989;10:236-44.

11. Didisheim P, Olsen DB, Fara DJ, et al. Infection and thromboembolism with implantable cardiovascular devices. Trans Am Soc Artif Intern Organs 1987;33:418-25.

12. Wagner WR, Johnson PC, Kormos RL, Griffith P. Evaluation of bioprosthetic valve-associated thrombus in ventricular assist device patients. Circulation 1993;88(Suppl):I2023-9.

13. Bernhard WF, LaFarge CG, Robinson TC, Shirahige K. An improved blood pump interface for left ventricular bypass. Ann Surg 1968;168:750.

14. Clark R, Boyd J, Moran J. New principles governing the tissue reactivity of prosthetic materials. J Surg Res 1974;16: 510-22.

15. Dasse KA, Chipman S, Sherman C, Levine AH, Frazier OH. Clinical experience with textured blood contacting surfaces in ventricular assist devices (VADs). Trans Am Soc Artif Intern Organs 1987;33:418-25.

16. Rose EA, Levin $\mathrm{HR}, \mathrm{Oz} \mathrm{MC}$, et al. Artificial circulatory support with textured interior surfaces: a counterintuitive approach to minimize thromboembolism. Circulation 1994; 90(5 Pt 2):II87-91.

17. Graham TR, Dasse K, Coumbe A, et al. Neointimal development on textured biomaterial surfaces during clinical use of an implantable left ventricular assist device. Eur $\mathbf{J}$ Cardiothoracic Surg 1990;4:182-90.

18. Slater JP, Rose EA, Levin HR, Frazier $\mathrm{OH}$, Roberts JK, Oz MC. Low thromboembolic risk without anticoagulation using advanced design left ventricular assist devices. Ann Surg. In press.

19. Bauer KA, Broekmans AW, Bertina RM, Conrad J, Horellou MH, Samama MM, et al. Hemostatic enzyme generation in the blood of patients with hereditary protein $\mathrm{C}$ deficiency. Blood 1988;71:1418-26.

20. Teitel JM, Bauer KA. Lau HK, Rosenberg RD. Studies of the prothrombin activation pathway utilizing radioimmuno- assays for the $F_{2} / F_{1+2}$ fragment and thrombin-antithrombin complex. Blood 1992;59:1086-97.

21. Kruskal WH, Wallis WA. Use of ranks on one-criterion variance analysis. J Am Stat Assoc 1952; 47:583-621.

22. Conover WJ. Practical nonparametric statistics. New York: John Wiley, 1980.

23. Fleiss JL. The design and analysis of clinical experiments. New York: John Wiley, 1986.

24. Yaqoob M, Patrick AW, McClelland P, et al. Relationship between markers of endothelial dysfunction, oxidant injury and tubular damage in patients with insulin-dependent diabetes mellitus. Clin Sci 1993;85:557-62.

25. Livingston ER, Fisher CA, Pathak AS, et al. Increased activation of the coagulation and fibrinolytic systems in left ventricular assist device recipients may lead to hemorrhagic complications. Circulation 1995;92:P83.

26. Salih V, Berry CL, Smith SC, et al. The lining of textured surfaces in implantable left ventricular assist devices: an immunocytochemical and electronmicroscopic study. Am J Cardiovasc Pathol 1993;4:317-25.

27. Frazier OH, Baldwin RT, Eskin SG, Duncan JM. Immunochemical identification of human endothelial cells on the lining of a ventricular assist device. Texas Heart Inst J 1993;20:78-82.

28. Menconi MJ, Owen T, Dasse KA, Stein G, Lian JB. Molecular approaches to the characterization of cell and blood/ biomaterial interactions. J Cardiac Surg 1992;7:177-87.

29. Menconi MJ, Prockwinse S, Owen TA, Dasse KA, Stein GS, Lian JB. Properties of blood-contacting surfaces of clinically implanted cardiac assist devices: gene expression, matrix composition, and ultrastructural characterization of cellular linings. J Cell Biochem 1995;57:557-73.

30. Rafii S, Oz MC, Seldomridge JA, et al. Characterization of human hematopoietic cells arising on the textured surface of left ventricular devices. Ann Thorac Surg 1995;60:162732. 